\title{
On the design and selection of vehicle coordination policies for underground mine production ramps
}

\author{
Michal Pasternak ${ }^{\mathrm{a}}$, Joshua A. Marshall ${ }^{\mathrm{a}, *}$ \\ ${ }^{a}$ Mining Systems Laboratory, The Robert M. Buchan Department of Mining, \\ Queen's University, Kingston ON K7L 3N6 Canada
}

\begin{abstract}
Traffic management in underground mines, especially on production ramps, is a difficult problem to optimize and control. Most operations use one of a few common policies; e.g., the so-called "lock-out" and "loaded-vehiclepriority" policies. The work presented in this paper uses discrete-event simulation to study the efficiency of multiple policies. Based on simulation results, an improvement to the common lock-out policy is proposed. This new policy utilizes the rules of the lock-out policy but integrates an option that allows a group of vehicles to be given temporary priority in a certain direction of travel. Quantitative results are provided and, based on these, a systematic technique for ramp design that aims to optimize the efficiency of underground mine ramp traffic flow is described.
\end{abstract}

Keywords: underground mining, fleet management, ramp design, equipment selection, discrete-event simulation

\section{Introduction}

This paper explores traffic management policies commonly used in underground mining operations, quantifies the parameters of the widely used "lock-out" policy and suggests a new, alternative policy. The lock-out policy is used as a basis for optimizing the preliminary design of a haulage ramp, and the selection of a haulage fleet for an underground mine production ramp.

The operating environment in underground mines is usually dark, dusty, and visibility is poor. Traffic can be a problem in mines where ore is hauled by truck or LHD directly to a crusher, conveyor belt, or up to surface $[4,7]$. In underground mines, ramps and passageways that are used for haulage are only wide enough to accommodate traffic in one direction at a time. However, to maximize productivity while keeping mining costs low, these ramps must also be capable of coordinating bi-directional traffic of vehicles. This situation introduces an added constraint over the dispatch problem in open pit mines [5]. This problem can also be

\footnotetext{
${ }^{*}$ Corresponding author.

Email addresses: michal.z.pasternak@queensu.ca

(Michal Pasternak), joshua.marshall@queensu. ca (Joshua A. Marshall)

URL: https ://msl.engineering . queensu . ca (Joshua A. Marshall)
}

Preprint submitted to Int. J. Min. Sci. Tech. observed in a wide variety of other industries such as examined by [2] and [13].

Production and service vehicles usually travel along the ramp at different speeds, are hard to stop, and are often loaded with materials. Short drifts, long enough to accommodate one vehicle, serve as passing bays. These drifts are left along the length of the ramp; e.g., see Fig. 1. Mine levels are commonly used as well for passing. The typically long ramp length and dispersed passing bays, together with varying traffic densities, can greatly increase the total travel times for production vehicles on the ramp. This combination of factors can, in some cases, also lead to serious safety concerns.

Based on our own experience at that of our industry partners, in uncontrolled ramps the interaction of two groups of vehicles is one of the leading causes of time loss and safety issues. The least desirable interaction occurs when groups that are travelling in opposite directions meet on a ramp segment, possibly far from a passing bay or level. To resolve the situation, one of the groups must reverse back to the closest vehicle-passing bay. A complicated back-and-forth manoeuvre is often required to allow one group of vehicles to pass the other, one at a time if the bay is only large enough for one vehicle. It can be a frustrating process because each time only one vehicle can pass the group at the passing bay, meaning that the group must move back and forth around the passing bay until each vehicle in the

July 27, 2015 


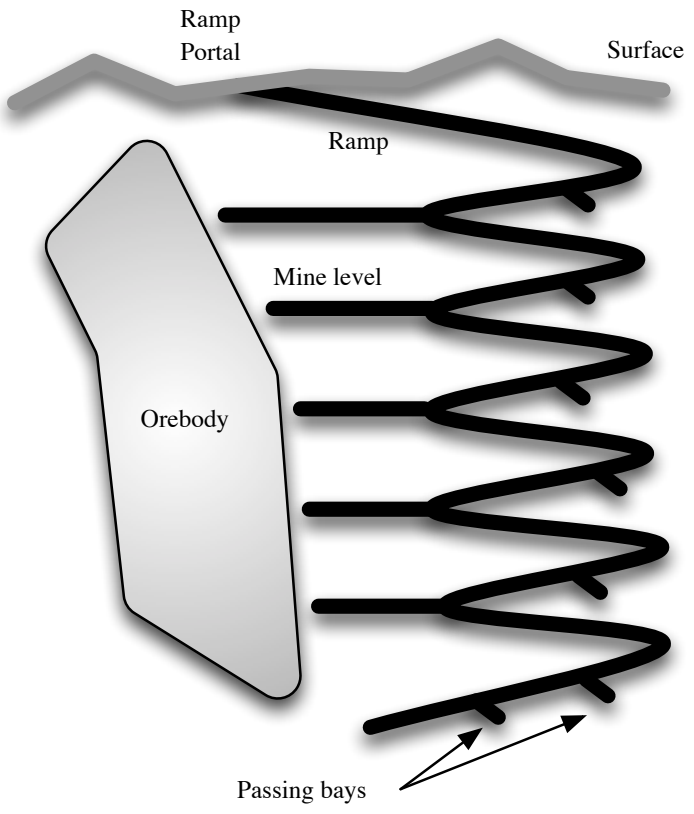

Fig. 1. Schematic drawing of a typical mine ramp-based underground haulage system, after [7].

other group has been passed. Much time, fuel and operator patience is lost in this manoeuvre. This type of vehicle interaction is a problem that must be completely avoided when implementing a haulage ramp management plan.

\subsection{Background}

Optimization of mining processes has been researched since the early 1960s [9]. Early on, optimization and vehicle dispatching was accomplished by using heuristic algorithms to navigate the tight underground environment [6]. Today vehicle dispatching is acknowledged as an essential optimization tool in mining and other industries and dispatch systems are installed on a wide variety of machines $[17,3]$.

However, satellite-based GPS tracking cannot be used for vehicle dispatching purposes in underground mining, which makes it difficult for modern dispatching systems to be of use [3]. New technologies under development show promise to overcome this problem. For example, map-based localization techniques from robotics have recently been a focus of research and development activities $[8,10]$. Real time position data on vehicles underground would allow for continuous monitoring and optimization of the system. Such a position monitoring system could shorten overall vehicle travel times when a large number of vehicles are present on the ramp, as shown by [16].

Discrete-event simulation can be used to study and optimize a wide variety of problems that involve objects moving along paths to various nodes, as shown by [2, $14,17]$. It is also one of the most popular tools used in solving other vehicle dispatch problems encountered in underground mines $[9,7]$. Discrete-event simulation has been used for solving fleet management problems in a mine level [1]. With the availability of large amounts of computing power, today complex simulations can be run in a very practical amount of time.

Ramp design is an important aspect in the feasibility process for underground mining [12]. The ramp represents a very large capital cost and, in many cases, is critical for operations. As such, design optimization is warranted to minimize costs, maximize productivity, and to ensure accurate cost estimates for project feasibility studies as described by [15]. However, there are relatively few studies about ramp optimization for the purposes of improving vehicle traffic and throughput. On the other hand, much work has been done on equipment selection for a pre-set ramp design; e.g., see [11].

\subsection{About this Paper}

In this paper, discrete-event simulation software (Simul8) is used to simulate an underground haulage ramp. Policies that are commonly used in mining were tested in the simulator, and the results obtained were used to parameterize each policy. The so-called lockout policy was found to be the most effective policy in terms of productivity. An alternate policy is also proposed, which improves the efficiency of the lock-out policy during times of increased traffic. This new policy was then tested on a fixed ramp length, with varying vehicle and passing bay numbers. Based on these results, a technique is presented to allow for easy ramp and fleet design, with focus on optimization of productivity.

\section{Mine Ramp Traffic Management Policies}

At present, there are three types of ramp traffic management policies that are most commonly used in underground mines across Canada and around the world. These three policies are:

1. simple routing;

2. loaded priority; and,

3. lock-out.

The simple routing policy is a radio-based communications policy that is suitable for low-volume traffic. It 
relies on drivers to communicate over radio when they enter certain ramp segments, thus helping to avoid situations where other drivers enter these segments, possibly resulting in a traffic conflict, lost time, or an accident. The remaining two policies are discussed next; see also [12]. In the current paper, the lock-out policy is examined in detail, and a fourth solution, which we demonstrate enhances the lock-out policy, is offered.

\subsection{Loaded Priority}

The loaded priority policy allows loaded vehicles (i.e., those hauling ore or waste) to continue up the ramp without stopping at passing bays. Vehicles which are empty and travelling downwards are expected to keep out of the way of these vehicles by use of the passing bays. This policy is inefficient with higher volumes of traffic, and especially when the loading time of a vehicle is shorter than that of any ramp segment (this is easily shown by simulation or in practice). Intuitively, if the loading time is shorter than travel time on a ramp segment, vehicles will tend to bunch up at the dumping point, as entrance onto the ramp will be difficult with a high number of vehicles travelling upwards. This policy promotes grouping of vehicles, which results in long lines at the loading and dumping points and periods of time in which there are no vehicles either at the loading or dumping points.

\subsection{Lock-out Policy}

The lock-out system is relatively common because it is easy to implement in a mine haulage ramp. All that is needed is a set of traffic lights, which can be manually changed through the use of a pull rope. The lock-out system works by only allowing one vehicle to travel on each ramp segment, and requires that vehicles wait until the segment is "free" before entering it, regardless of whether the vehicle is travelling in the opposite direction or not. This system has some inherent inefficiencies, as vehicles which could travel together cannot according to the lock-out policy rules. When manually driven, human errors can also result in efficiencies.

\subsection{Simulator Implementation and Parameters}

To study and test the performance of rampmanagement policies, a model haulage ramp was created using a commercial discrete-event simulator software called Simul $8^{1}$. The software works by allowing the user to create a system of simulation "objects" that

\footnotetext{
${ }^{1}$ Simul8 Corporation: http://www . simul8. com/.
}

can modify and route other "work objects" throughout the system. This allows for the simulation of many vehicles (i.e., work objects) on the same ramp circuit.

To simulate bidirectional traffic in a single-lane passage, two parallel routes were created in Simul8, and a check was implemented to indicate a conflict if vehicles were present in both routes at the same time. A simple spreadsheet was used to keep track of vehicle positions on each ramp segment. If a crash was detected, the simulation was stopped, and a violation of the policy was indicated. The spreadsheets were updated at each discrete event to catch any possible violations of the policy.

To test one policy or variation against a different policy, an exact copy of the ramp was made and the logic of one of the duplicated ramp systems was replaced with that of the other policy to be studied. The ramp system was also designed in such a way that the incorporation of randomness in timing, other disturbances, as well as changes in traffic availability can be modelled. For this study, a ramp model that consisted of a single loading point, a single dump point, and seven ramp segments, each spaced with one passing bay was created for testing between policies. These parameters were kept fixed in order to see the effect of varying only policy, in the absence of other factors (i.e., the focus here is on policy design, not ramp layout design).

\section{Analysis of the Lock-Out Policy}

The lock-out policy works by allowing a vehicle to reserve a specified segment of ramp by pulling a string before entry into the area. This prohibits any other vehicles from entering the currently-reserved segment from either direction of the ramp. Once the vehicle has crossed through the reserved area, another string is pulled to release the segment. At this moment, the first waiting vehicle to pull the string for the free area is allowed to reserve the segment for travel. The lock-out policy is similar to a traffic light at a sparsely used location. When a vehicle requires access to a segment that is free it can easily reserve the segment and be sure that there will not be any interfering traffic in the segment. See also the work of [7] for a study related to the fundamental behaviours of ramp traffic under the lock-out policy.

\subsection{Algorithm}

The lock-out policy can be analyzed mathematically when some assumptions are made as to its implementation. The first being that the policy is implemented on the entirety of the haulage ramp and that each point 
at which vehicles pass from one segment to the next has a passing bay that can accommodate one vehicle. The policy can be implemented on a closed loop that includes a loading point and dumping point, where vehicles go back and forth between. Or, it can be implemented an open loop where vehicles enter and exit at the top and bottom of the ramp, and do not necessarily need to make a return trip right after reaching either the bottom or top of the ramp.

For haulage ramps, using the lock-out policy in closed loop, the maximum moving traffic is equal to the number of ramp segments plus one vehicle at both the loading and dumping point. If capacity at the loading and dumping points were to be greater than one vehicle each, this would not increase the maximum moving traffic in the system because only one vehicle is allowed to leave the loading and dumping point at a time. The only result would be increased waiting time and decreased loading and dumping time. This relationship can be expressed by

$$
M T=R S+2,
$$

where $M T$ is the maximum effective moving traffic and $R S$ is equivalent to the number of ramp segments in the system.

When analyzing an open system, the maximum moving traffic within that system is only equal to the number of segments in the system. This shows that, at a certain point, the addition of another production vehicle does not result in an increase in productivity.

\subsection{Observations from Simulation of the Lock-Out Pol- icy}

The maximum moving traffic number $M T$ corresponds to the point at which adding production vehicles to the system no longer increases the productivity of the system. This number represents an upper bound to the amount of traffic that can be handled by the ramp, and thus caps the production that can be reached with the lock-out policy for a given ramp design. This is shown by the simulation results in Fig. 2 for simulation time units required to achieve 200 loads.

The lock-out policy does a good job of spreading vehicles out along the ramp system. This promotes shorter queueing times and a more continues feed to the dump. The lock-out system is also efficient in that it eliminates many hazardous vehicle interactions, increasing safety on the ramp. The situation explained in the introduction, where groups meet at intersections, is one of the most inefficient and dangerous interactions and it is avoided when using the lock-out policy.
It was observed that a ramp system with a quantity of vehicles over the maximum moving traffic number $M T$ will result in a situation where vehicles tend to group and wait for entry onto the ramp. The location of these groupings can change depending on the initial distribution of the vehicles, as well as the time required for travelling on the ramp segments, and the loading and dumping times. The locations where grouping can occur are always immediately before the loading point, right after the loading point, and before and after the dumping point.

In ramps with ramp segments that take less time to traverse than the time required for the vehicle to load or unload at the loading or dumping point, the vehicles tend to not occupy every single ramp segment. They become spaced out in equivalence with the time required at the loading or dumping point and tend to form a line before the loading or dumping points. On ramps with ramp segments that take longer to traverse, a line tends to form after the loading or dumping points and before the entrance onto the ramp. For optimal dispersion of vehicles in the system, we recommended that practitioners attempt to match loading and unloading times, if possible, to the average time to traverse each ramp segment when operating at or close to the maximum moving traffic number.

\subsection{Limitations of the Basic Lock-Out Policy}

The lock-out policy reaches a traffic limit, at which point adding more vehicles to the system does not result in an increase of production. This can be seen in Fig. 2, once the number of vehicles exceeds the maximum traffic number (i.e., in this simulation, $M T=R S+2=9$ ), the simulation time units required to deliver 200 loads no longer improves. This is a major limitation to the performance of the ramp, as there may only be certain time periods at which a large number of vehicles are required to travel in one direction. It would be very time consuming to follow the lock-out policy to the letter in a case like this.

\section{Proposed Group Lock-Out Policy}

To further increase the productivity of existing infrastructure, a change in the lock-out policy is needed to increase the maximum moving traffic value. The aforementioned phenomenon of grouping, which occurs at certain parts of the ramp system, can be exploited by modifying the lock-out policy to allow certain groups to pass, if one occurs. When a group of specific size forms, the policy is changed to allow that group of vehicles to 


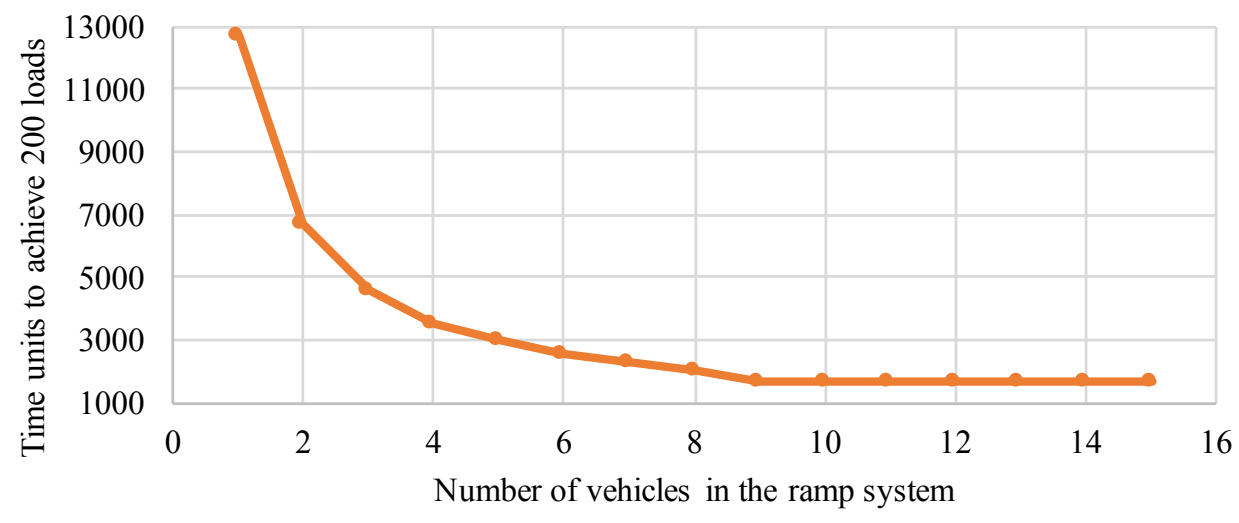

Fig. 2. Productivity of the basic lock-out policy for $R S=7$.

continue down the ramp as one unit. Since passing bays can only accommodate one vehicle at a time, vehicles travelling in the direction opposite to that of the group must be the ones using the passing bay.

Before a group enters a ramp segment it must first wait for the segment to be unlocked, as per the lockout policy. Instead of entering a passing bay, the group simply waits in the current ramp segment until the one ahead is cleared before continuing on. If the group does not clear the current ramp segment and the group has already started moving into the next ramp segment, the vehicle travelling in the opposite direction must wait in the passing bay until the entire group has passed. When the group starts moving into the next ramp segment, no new vehicles can latch on to the group, as this would further increase the waiting time of the vehicle travelling in the opposite direction. Only one group is allowed to travel on the ramp at a time.

These modifications to the basic lock-out policy give priority to all vehicles travelling in the direction of the group. Once the group has reached the other side of the ramp system, the policy reverts to the basic lock-out policy. When a group of specific size forms once again, priority can be given to the direction of travel associated with this new group.

\subsection{Simulation Results and Comparisons}

This policy effectively increases the maximum moving traffic of the ramp to include that of the travelling group. The new equation,

$$
M T=R S+2+G S
$$

includes $G S$, the group size, at which a group is deemed large enough to be given priority in its direction of travel.

The group size that can be used is limited by the total number of vehicles in the system. Since the lock-out policy spreads the vehicles out over the ramp system, the maximum group size that can form, $G S_{\text {max }}$, is given by $T T$, the total traffic in the system less $M T$, the maximum moving traffic, as in

$$
G S_{\max }=T T-M T .
$$

The size of the group that triggers a change in direction and lets the group pass does not have to be equal to the maximum group size. Smaller group sizes result in more frequent changes in directional priority, and can even result in the same direction being given priority many times over. By using larger group sizes priority is given less often, and vehicles are allowed to queue for longer amounts of time before being sent down as a group. Increasing group size may not necessarily improve productivity of the ramp system.

The performance of this modification to the lock-out policy can be seen in Fig. 3. As before, this system has $M T=9$ because $R S=7$ was used. Both policies tested, used identical parameters during the simulation. The $M T$ point can be clearly identified in Fig. 3, as it is the point where production stops improving with an increase in the number of vehicles. The grouping policy works exactly the same as the lock-out policy, however it is not bound by the same $M T$ value, and can increase productivity past the MT point.

\section{Group Lock-Out Policy and Ramp Design}

Overall ramp length is often a fixed parameter when it comes to designing a ramp, because grade and depth 


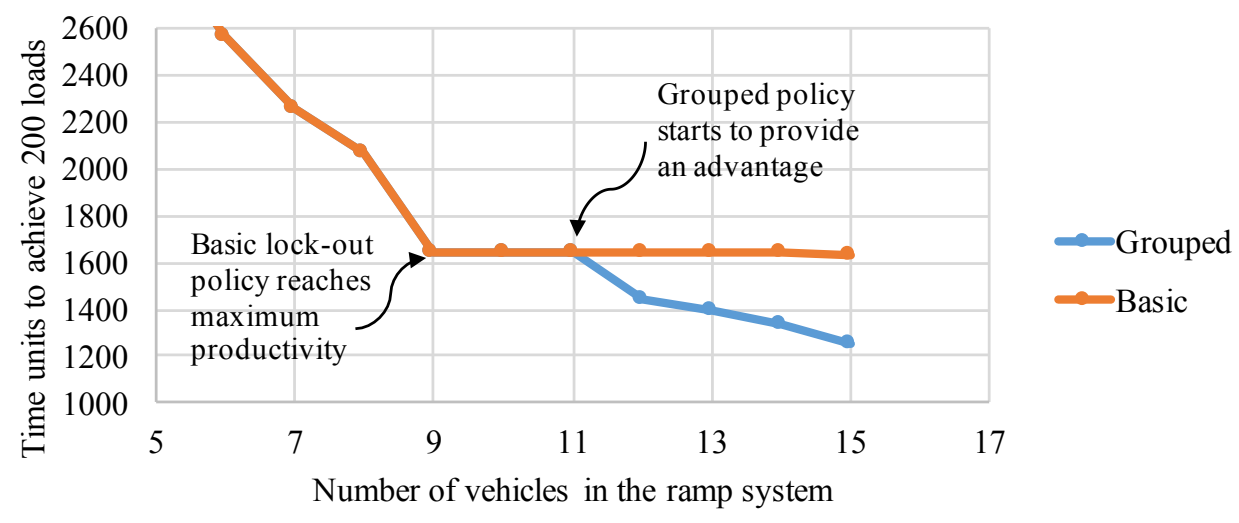

Fig. 3. Performance comparison of lock-out versus the grouped lock-out policy

is optimized using specialized software [15]. The slope and depth are dictated by the type of ore body, as well as vehicle performance and safety. The number of passing bays on a haulage ramp is often decided as a rule of thumb, with little optimization effort being given to the amount of bays on the ramp. Maximizing the achievable production from a ramp is an important task; in some mines it is the only way of moving ore to surface. From the analysis of traffic policies presented earlier in this paper, we know that the maximum moving traffic number and the number of vehicles dictate the production capacity of a haulage ramp. Increasing maximum moving traffic number means a larger ramp system capacity, and thus a higher production rate.

The moving traffic number is primarily affected by the number of ramp segments in the ramp system. The more passing bays on the ramp, the more segments there are and the higher the maximum moving traffic number. When vehicle number is increased, production rate increases. For certain combinations, the returns on extra vehicles or from adding an extra passing bay become minimal or non-existent. Thus, when designing a ramp it is useful to know in which direction to move the design that maximizes productivity and traffic flow.

When designing a ramp, the traffic on the ramp, and the targeted production rate play an important role in determining the number of passing bays. All these factors must be known before starting construction of the ramp in order to optimize the system. By running simulations and varying the number of passing bays, and vehicles on a ramp of fixed length, the optimal balance of passing bays and vehicles for a given policy and ramp length can be estimated.

By simulating many combinations of vehicle numbers and numbers of passing bays, we can provide a tool by which mine designers might optimize productivity. The example simulation results of Fig. 4 shows the average time required to complete 200 cycles on the ramp. Should a passing bay or vehicle be added to improve productivity? To quickly illustrate which option is most advantageous, a flow diagram, showing the path of greatest return is provided in Figure 5. This design chart shows in which direction the optimal design step would be. For example, for a current design with 7 vehicles and 5 passing bays, one would be better off increasing the number of passing bays (i.e., "right" on the chart). For a design with 5 vehicles and 3 passing bays, one should make no incremental design changes (i.e., "stay" on the chart).

The method can be used for any policy that is to be implemented in the mine. By simulating the policy (e.g., in Simul8 or some other discrete-event software tool), predicted efficiency of the ramp system can be measured numerically and a direct comparison can be made between policies or between different ramp designs and fleet configurations.

\section{Conclusion}

1. Under fundamental assumptions (e.g., no random vehicle presence, single load-dump points, other random disturbances), the presented research shows how the common lock-out policy for underground ramp management can be modified to increase the traffic flow on mine production ramps in a way that does not increase the number of (undesirable) vehicle encounters.

2. Simulation results using a commercial simulation tool show how there is a productivity limit that depends on the number of ramp segments available. 


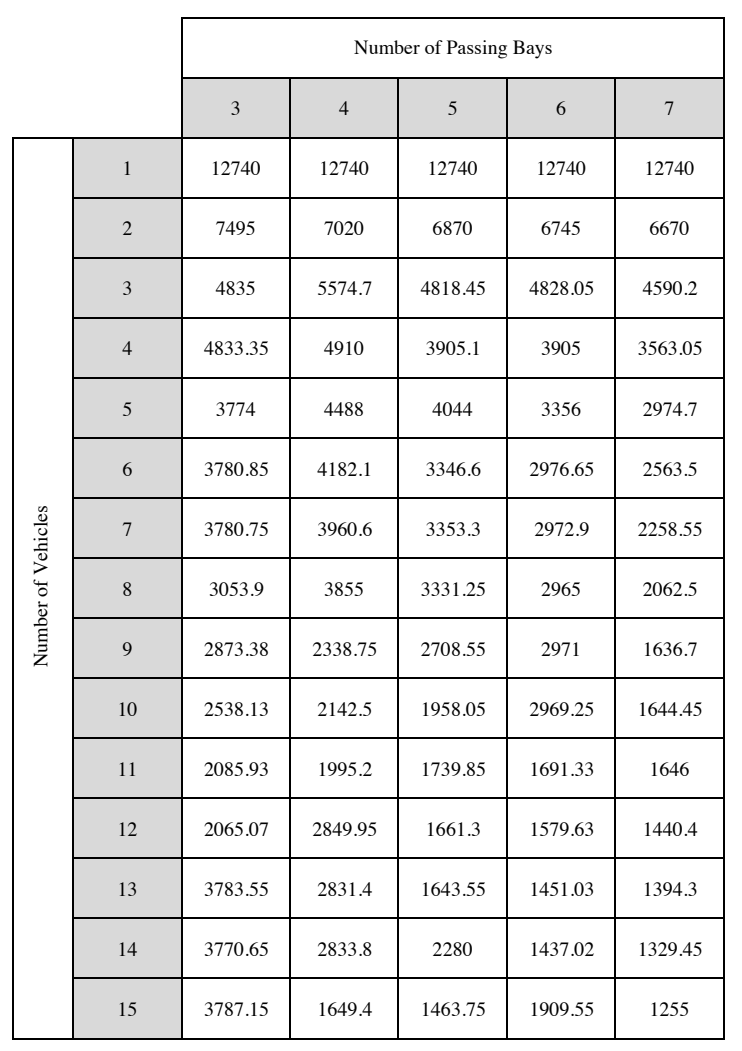

Fig. 4. Time units to 200 cycles for various combinations of number vehicles in the ramp system and number of passing bays, illustrating how incremental changes affect productivity.

\begin{tabular}{|c|c|c|c|c|c|c|}
\hline & & \multicolumn{5}{|c|}{ Number of Passing Bays } \\
\hline & & 3 & 4 & 5 & 6 & 7 \\
\hline \multirow{15}{*}{ 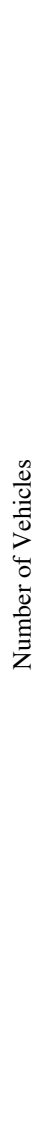 } & 1 & down & down & down & down & down \\
\hline & 2 & down & down & down & down & down \\
\hline & 3 & down & right & down & down & down \\
\hline & 4 & down & right & right & down & down \\
\hline & 5 & stay & left & down & right & down \\
\hline & 6 & up & right & right & right & down \\
\hline & 7 & down & right & right & right & down \\
\hline & 8 & down & down & down & right & down \\
\hline & 9 & right & down & down & right & stay \\
\hline & 10 & down & right & down & right & up \\
\hline & 11 & right & right & down & down & down \\
\hline & 12 & stay & right & right & right & down \\
\hline & 13 & up & right & right & right & down \\
\hline & 14 & right & down & right & right & down \\
\hline & 15 & right & right & stay & right & stay \\
\hline
\end{tabular}

Fig. 5. Design map showing incremental path of greatest gain (i.e., how to change passing bays and/or number of vehicles); note the existence of local maxima at certain design points, indicating that (locally) changing the number of passing bays or vehicles in the system is not beneficial. 
3. For optimal dispersion of vehicles in a lock-out based system, we recommended that practitioners attempt to match loading and unloading times, if possible, to the average time to traverse each ramp segment when operating at or close to the maximum moving traffic number.

4. By using a modified lock-out policy, which enables occasional grouping, a greater productivity can be reached with the same level of safety.

5. We show how ramp design can be conducted based on the desired policy, and optimized for the policy to achieve the highest vehicle throughput and ramp productivity. By performing a sequence of simulations, a design map can be produced for optimizing design aspects of a ramp for a given policy. The presented method could be used to test any policy on a chosen ramp design.

\section{Acknowledgements}

This project was in part funded by the Natural Science and Engineering Research Council of Canada (NSERC) under project CRDPJ 44SBO4-12, and by funding provided by Barrick Gold Corporation and Peck Tech Consulting Ltd. Thanks to Graham Hanson of Barrick Gold Corporation and Andrew Chapman of Peck Tech Consulting Ltd. for providing information used to help in modelling of the haulage ramp used for testing policies.

\section{References}

[1] Beaulieu, M., Gamache, M., 2006. An enumeration algorithm for solving the fleet management problem in underground mines. Computers \& Operations Research 33 (6), 1606-1624.

[2] Bussieck, M. R., Winter, T., Zimmermann, U. T., January 1997. Discrete optimization in public rail transport. Mathematical Programming 79 (1-3), 415-444.

[3] Cantin, S., February 2011. Under control: Keeping track of what's happening underground. Canadian Mining Journal $132(2)$.

[4] Gamache, M., Cohen, P., Grimard, R., Bigras, L., February 2004. Fleet management system for underground mines. CIM Bulletin 97 (1077), 66-70.

[5] Gamache, M., Grimard, R., Cohen, P., October 2005. A shortestpath algorithm for solving the fleet management problem in underground mines. European Journal of Operational Research 166 (2), 497-506.

[6] Gillett, B., Miller, L., March-April 1974. A heuristic algorithm for the vehicle-dispatch problem. Operations Research 22 (2), 340-349

[7] Haviland, D., Marshall, J. A., January 2015. Fundamental behaviours of production traffic in underground mine haulage ramps. International Journal of Mining Science and Technology 25 (1), 7-14.
[8] Lavigne, N. J., Marshall, J. A., November/December 2012. A landmark-bounded method for large-scale underground mine mapping. Journal of Field Robotics 29 (6), 861-879.

[9] Newman, A. M., Rubio, E., Caro, R., Weintraub, A., Eurek, K., May 2010. A review of operations research in mine planning. Interfaces 40 (3), 222-245.

[10] Radacina-Rusu, S., Marshall, J. A., Hayes, M. J. D., May 2011. Localization in large-scale underground environments with RFID. In: Proceedings of the 24th IEEE Canadian Conference on Electrical and Computer Engineering (CCECE). IEEE, Niagara Fall, ON, pp. 1140-1143.

[11] Salama, A., Greberg, J., Schunnesson, H., 2014. The use of discrete event simulation for underground haulage mining equipment selection. International Journal of Mining and Mineral Engineering 5 (3), 256-271.

[12] Sturgul, J. R., Jacobsen, W. L., Tecsa, T. L., 1996. Modeling two-way traffic in an underground one-way decline. In: Mine Planning and Equipment Selection. Vol. W.T. Hennies and L.A. Ayres de Silva and A.P. Chaves. Balkema, pp. 87-90.

[13] Sun, T. C., Lai, K. K., Lam, K., So., K. P., January 1994. A study of heuristics for bidirectional multi-hoist production scheduling systems. International Journal of Production Economics 33 (13), 201-214.

[14] Tarshizi, E., Sturgul, J., Ibarra, V., Taylor, D., July 2015. Simulation and animation model to boost mining efficiency and envirofriendly in multi-pit operations. International Journal of Mining Science and Technology 25 (4), 2095-2686.

[15] Thomas, D. A., Brazil, M., Lee, D. H., Wormald, N. C., 2007. Network modelling of underground mine layout: two case studies. International Transactions in Operational Research 14 (2), 143-158.

[16] Vagenas, N., 1991. Dispatch control of a fleet of remotecontrolled/automatic load-haul-dump vehicles in underground mines. International Journal of Production Research 29 (11), 2347-2363.

[17] Winter, T., Zimmermann, U., 2000. Real-time dispatch of trams in storage yards. Annals of Operations Research 96 (1-4), $287-$ 315 . 